\title{
Some new nonlinear retarded sum-difference inequalities with applications
}

\author{
Wu-Sheng Wang ${ }^{1 *}$, Zizun $\mathrm{Li}^{2}$ and Wing-Sum Cheung ${ }^{3}$
}

* Correspondence: wang4896@126. com

'Department of Mathematics, Hechi University, Guangxi, Yizhou 546300, People's Republic of China Full list of author information is available at the end of the article

\section{Abstract}

The main objective of this paper is to establish some new retarded nonlinear sumdifference inequalities with two independent variables, which provide explicit bounds on unknown functions. These inequalities given here can be used as handy tools in the study of boundary value problems in partial difference equations. 2000 Mathematics Subject Classification: 26D10; 26D15; 26020.

Keywords: sum-difference inequalities, boundary value problem

\section{Introduction}

Being important tools in the study of differential, integral, and integro-differential equations, various generalizations of Gronwall inequality [1,2] and their applications have attracted great interests of many mathematicians (cf. [3-16], and the references cited therein). Recently, Agarwal et al. [3] studied the inequality

$$
u(t) \leq a(t)+\sum_{i=1}^{n} \int_{b_{i}\left(t_{0}\right)}^{b_{i}(t)} g_{i}(t, s) w_{i}(u(s)) d s, \quad t_{0} \leq t<t_{1} .
$$

Cheung [17] investigated the inequality

$$
\begin{aligned}
u^{p}(x, y) \leq & a+\frac{p}{p-q} \int_{b_{1}\left(x_{0}\right)}^{b_{1}(x)} \int_{c_{1}\left(y_{0}\right)}^{b_{1}(y)} g_{1}(s, t) u^{q}(s, t) \mathrm{d} t \mathrm{~d} s \\
& +\frac{p}{p-q} \int_{b_{2}\left(x_{0}\right)}^{b_{2}(x)} \int_{c_{2}\left(y_{0}\right)}^{c_{2}(y)} g_{2}(s, t) u^{q}(s, t) \psi(u(s, t)) \mathrm{d} t \mathrm{~d} s .
\end{aligned}
$$

Agarwal et al. [18] obtained explicit bounds to the solutions of the following retarded integral inequalities: 


$$
\begin{aligned}
& \varphi(u(t)) \leq c+\sum_{i=1}^{n} \int_{\alpha_{i}\left(t_{0}\right)}^{\alpha_{i}(t)} u^{q}(s)\left[f_{i}(s) \varphi(u(s))+g_{i}(s)\right] \mathrm{d} s, \\
& \varphi(u(t)) \leq c+\sum_{i=1}^{n} \int_{\alpha_{i}\left(t_{0}\right)}^{\alpha_{i}(t)} u^{q}(s)\left[f_{i}(s) \varphi_{1}(u(s))+g_{i}(s) \varphi_{2}(\log u(s))\right] \mathrm{d} s, \\
& \varphi(u(t)) \leq c+\sum_{i=1}^{n} \int_{\alpha_{i}\left(t_{0}\right)}^{\alpha_{i}(t)} u^{q}(s)\left[f_{i}(s) L(s, u(s))+g_{i}(s) u(s)\right] \mathrm{d} s,
\end{aligned}
$$

where $c$ is a constant, and Chen et al. [19] did the same for the following inequalities:

$$
\begin{aligned}
\psi(u(x, y)) \leq & c+\int_{\gamma\left(x_{0}\right)}^{\gamma(x)} \int_{\delta\left(y_{0}\right)}^{\delta(y)} f(s, t) \varphi(u(s, t)) \mathrm{d} t \mathrm{~d} s, \\
\psi(u(x, y)) \leq c+ & \int_{\alpha\left(x_{0}\right)}^{\alpha(x)} \int_{\beta\left(y_{0}\right)}^{\beta(y)} g(s, t) u(s, t) \mathrm{d} t \mathrm{~d} s \\
& +\int_{\gamma\left(x_{0}\right)}^{\gamma(x)} \int_{\delta\left(y_{0}\right)}^{\delta(y)} f(s, t) u(s, t) \varphi(u(s, t)) \mathrm{d} t \mathrm{~d} s, \\
\psi(u(x, y)) \leq & c+\int_{\alpha\left(x_{0}\right)}^{\alpha(x)} \int_{\beta\left(y_{0}\right)}^{\beta(y)} g(s, t) w(u(s, t)) \mathrm{d} t \mathrm{~d} s \\
& +\int_{\gamma(x)} \int_{\gamma\left(x_{0}\right)}^{\delta(y)} f(s, t) w(u(s, t)) \varphi(u(s, t)) \mathrm{d} t \mathrm{~d} s,
\end{aligned}
$$

where $c$ is a constant.

Along with the development of the theory of integral inequalities and the theory of difference equations, more attentions are drawn to some discrete versions of Gronwall type inequalities (e.g., [20-22] for some early works). Some recent works can be found, e.g., in [6,23-25] and some references therein. Found in [26], the unknown function $u$ in the fundamental form of sum-difference inequality

$$
u(n) \leq a(n)+\sum_{s=0}^{n-1} f(s) u(s)
$$

can be estimated by $u(n) \leq a(n) \prod_{s=0}^{n-1}(1+f(s))$. In [6], the inequality of two variables

$$
u^{2}(m, n) \leq c^{2}+\sum_{s=m_{0}}^{m-1} \sum_{t=n_{0}}^{n-1} a(s, t) u(s, t)+\sum_{s=m_{0}}^{m-1} \sum_{t=n_{0}}^{n-1} b(s, t) u(s, t) w(u(s, t))
$$


was discussed, and the result was generalized in [23] to the inequality

$$
u^{p}(m, n) \leq c+\sum_{s=m_{0}}^{m-1} \sum_{t=n_{0}}^{n-1} a(s, t) u^{q}(s, t)+\sum_{s=m_{0}}^{m-1} \sum_{t=n_{0}}^{n-1} b(s, t) u^{q}(s, t) w(u(s, t)) .
$$

In this paper, motivated mainly by the works of Cheung [17,23], Agarwal et al. [3,18], and Chen et al. [19], we shall discuss upper bounds of the function $u(m, n)$ satisfying one of the following general sum-difference inequalities

$$
\begin{aligned}
& \psi(u(m, n)) \leq a(m, n)+b(m, n) \sum_{i=1}^{k} \sum_{s=m_{0}}^{m-1} \sum_{t=n_{0}}^{n-1} w\left(u\left(\alpha_{i}(s), \beta_{i}(t)\right)\right)\left[f_{i}(s, t) \varphi\left(u\left(\alpha_{i}(s), \beta_{i}(t)\right)\right)\right. \\
& \left.+g_{i}(s, t)\right] \text {, } \\
& \psi(u(m, n)) \leq a(m, n)+b(m, n) \sum_{i=1}^{k} \sum_{s=m_{0}}^{m-1} \sum_{t=n_{0}}^{n-1} w\left(u\left(\alpha_{i}(s), \beta_{i}(t)\right)\right)\left[f_{i}(s, t) \varphi_{1}\left(u\left(\alpha_{i}(s), \beta_{i}(t)\right)\right)\right. \\
& \left.+g_{i}(s, t) \varphi_{2}\left(\log u\left(\alpha_{i}(s), \beta_{i}(t)\right)\right)\right], \\
& \psi(u(m, n)) \leq a(m, n)+b(m, n) \sum_{i=1}^{k} \sum_{s=m_{0}}^{m-1} \sum_{t=n_{0}}^{n-1} w\left(u\left(\alpha_{i}(s), \beta_{i}(t)\right)\right)\left[f_{i}(s, t) L\left(s, t, u\left(\alpha_{i}(s), \beta_{i}(t)\right)\right)\right. \\
& \left.+g_{i}(s, t) u\left(\alpha_{i}(s), \beta_{i}(t)\right)\right],
\end{aligned}
$$

for $(m, n) \in\left[m_{0}, m_{1}\right) \cap \mathbb{N}_{+} \times\left[n_{0}, n_{1}\right) \cap \mathbb{N}_{+}$, where $a(m, n), b(m, n)$ are nonnegative and nonde-creasing functions in each variable. Inequalities (1.1), (1.2), and (1.3) are the discrete versions of Agarwal et al. [18] and Chen et al. [19]. They not only generalized the forms with one variable into the ones with two variables but also extended the constant ' $c$ ' out of integral into a function ' $a(m, n)$ '. These inequalities will play an important part in the study on difference equation. To illustrate the action of their inequalities, we also gave an example of boundary value problem in partial difference equation.

\section{Main result}

Throughout this paper, $k, m_{0}, m_{1}, n_{0}, n_{1}$ are fixed natural numbers. $\mathbb{N}_{+}:=\{1,2,3, \ldots\}$, $I:=\left[m_{0}, m_{1}\right] \cap \mathbb{N}_{+}, I_{m}:=\left[m_{0}, m\right] \cap \mathbb{N}_{+}, J:=\left[n_{0}, n_{1}\right] \cap \mathbb{N}_{+}, J_{n}:=\left[n_{0}, n\right] \cap \mathbb{N}_{+}, \mathbb{R}_{+}:=[0$, $\infty)$. For functions $s(m), z(m, n), m, n \in \mathbb{N}$, their first-order (forward) differences are defined by $\Delta s(m)=s(m+1)-s(m), \Delta_{1} z(m, n)=z(m+1, n)-z(m, n)$ and $\Delta_{2} z(m, n)=$ $z(m, n+1)-z(m, n)$. Obviously, the linear difference equation $\Delta x(m)=b(m)$ with initial condition $x\left(m_{0}\right)=0$ has solution $\sum_{s=m_{0}}^{m-1} b(s)$. For convenience, in the sequel, we define $\sum_{s=m_{0}}^{m_{0}-1} b(s)=0$. We make the following assumptions:

$\left(H_{1}\right) \psi \in C\left(\mathbb{R}_{+}, \mathbb{R}_{+}\right)$is strictly increasing with $\psi(0)=0$ and $\psi(t) \rightarrow \infty$ as $t \rightarrow \infty$;

$\left(H_{2}\right) a, b: I \times J \rightarrow(0, \infty)$ are nondecreasing in each variable;

$\left(H_{3}\right) w, \phi, \phi_{1}, \phi_{2} \in C\left(\mathbb{R}_{+}, \mathbb{R}_{+}\right)$are nondecreasing with $w(0)>0, \phi(r)>0, \phi_{1}(r)>0$ and $\phi_{2}(r)>0$ for $r>0$;

$\left(H_{4}\right) \alpha_{i}: I \rightarrow I$ and $\beta_{i}: J \rightarrow J$ are nondecreasing with $\alpha_{i}(m) \leq m$ and $\beta_{i}(n) \leq n, i=1,2$, $\ldots, k$

$\left(H_{5}\right) f_{i}, g_{i}: I \times J \rightarrow \mathbb{R}_{+}, i=1,2, \ldots, k$.

Theorem 1. Suppose $\left(H_{1}-H_{5}\right)$ hold and $u(m, n)$ is a nonnegative function on $I \times J$ satisfying (1.1). Then, we have 


$$
u(m, n) \leq \psi^{-1}\left[W^{-1}\left(\Phi^{-1}(A(m, n))\right)\right]
$$

for all $(m, n) \in I_{M_{1}} \times J_{N_{1}}$, where

$$
\begin{aligned}
& W(r):=\int_{1}^{r} \frac{\mathrm{d} s}{w\left(\psi^{-1}(s)\right)} \text { for } r>0 ; \quad W(0):=\lim _{r \rightarrow 0^{+}} W(r), \\
& \Phi(r):=\int_{1}^{r} \frac{\mathrm{d} s}{\varphi\left(\psi^{-1}\left(W^{-1}(s)\right)\right)} \text { for } r>0 ; \quad \Phi(0):=\lim _{r \rightarrow 0^{+}} \Phi(r), \\
& A(m, n):=\Phi\left(W(a(m, n))+b(m, n) \sum_{i=1}^{k} \sum_{s=m_{0}}^{m-1} \sum_{t=n_{0}}^{n-1} g_{i}(s, t)\right)+b(m, n) \sum_{i=1}^{k} \sum_{s=m_{0}}^{m-1} \sum_{t=n_{0}}^{n-1} f_{i}(s, t),
\end{aligned}
$$

and $\left(M_{1}, N_{1}\right) \in I \times J$ is arbitrarily chosen such that

$$
A\left(M_{1}, N_{1}\right) \in \operatorname{Dom}\left(\Phi^{-1}\right), \Phi^{-1}\left(A\left(M_{1}, N_{1}\right)\right) \in \operatorname{Dom}\left(W^{-1}\right) .
$$

Proof. From assumption $\left(H_{2}\right)$ and the inequality (1.1), we have

$$
\begin{aligned}
\psi(u(m, n)) \leq & a(M, n)+b(M, n) \sum_{i=1}^{k} \sum_{s=m_{0}}^{m-1} \sum_{t=n_{0}}^{n-1} w\left(u\left(\alpha_{i}(s), \beta_{i}(t)\right)\right) \\
& \cdot\left[f_{i}(s, t) \varphi\left(u\left(\alpha_{i}(s), \beta_{i}(t)\right)\right)+g_{i}(s, t)\right]
\end{aligned}
$$

for all $(m, n) \in I_{M} \times J$, where $m_{0} \leq M \leq M_{1}$ is a natural number chosen arbitrarily. Define a function $\eta(m, n)$ by the right-hand side of (2.6). Clearly, $\eta(m, n)$ is positive and nondecreasing in each variable, with $\eta\left(m_{0}, n\right)=a(M, n)>0$. Hence (2.6) is equivalent to

$$
u(m, n) \leq \psi^{-1}(\eta(m, n))
$$

for all $(m, n) \in I_{M} \times J$. By $(H 4)$ and the monotonicity of $w, \psi^{-1}$ and $\eta$, we have, for all $(m, n) \in I_{M} \times J$,

$$
\begin{aligned}
\Delta_{1} \eta(m, n) & =b(M, n) \sum_{i=1}^{k} \sum_{t=n_{0}}^{n-1} w\left(u\left(\alpha_{i}(m), \beta_{i}(t)\right)\right)\left[f_{i}(m, t) \varphi\left(u\left(\alpha_{i}(m), \beta_{i}(t)\right)\right)+g_{i}(m, t)\right] \\
& \leq w\left(\psi^{-1}(\eta(m, n))\right) b(M, n) \sum_{i=1}^{k} \sum_{t=n_{0}}^{n-1}\left[f_{i}(m, t) \varphi\left(\psi^{-1}(\eta(m, t))\right)+g_{i}(m, t)\right] .
\end{aligned}
$$

On the other hand, by the monotonicity of $w$ and $\psi^{-1}$,

$$
W(\eta(m+1, n))-W(\eta(m, n))=\int_{\eta(m, n)}^{\eta(m+1, n)} \frac{\mathrm{d} s}{w\left(\psi^{-1}(s)\right)} \leq \frac{\Delta_{1} \eta(m, n)}{w\left(\psi^{-1}(\eta(m, n))\right)} .
$$

From (2.8) and (2.9), we have

$$
\begin{aligned}
& W(\eta(m+1, n))-W(\eta(m, n)) \\
& \quad \leq b(M, n) \sum_{i=1}^{k} \sum_{t=n_{0}}^{n-1}\left[f_{i}(m, t) \varphi\left(\psi^{-1}(\eta(m, t))\right)+g_{i}(m, t)\right]
\end{aligned}
$$


for ( $m, n),(m+1, n) \in I_{M} \times J$. Keeping $n$ fixed and substituting $m$ with $s$ in (2.10), and then summing up both sides over $s$ from $m_{0}$ to $m-1$, we get

$$
\begin{aligned}
W(\eta(m, n)) & \leq W\left(\eta\left(m_{0}, n\right)\right)+b(M, n) \sum_{i=1}^{k} \sum_{s=m_{0}}^{m-1} \sum_{t=n_{0}}^{n-1}\left[f_{i}(s, t) \varphi\left(\psi^{-1}(\eta(s, t))\right)+g_{i}(s, t)\right] \\
& =W(a(M, n))+b(M, n) \sum_{i=1}^{k} \sum_{s=m_{0}}^{m-1} \sum_{t=n_{0}}^{n-1}\left[f_{i}(s, t) \varphi\left(\psi^{-1}(\eta(s, t))\right)+g_{i}(s, t)\right] \\
& \leq c(M, n)+b(M, n) \sum_{i=1}^{k} \sum_{s=m_{0}}^{m-1} \sum_{t=n_{0}}^{n-1} f_{i}(s, t) \varphi\left(\psi^{-1}(\eta(s, t))\right)
\end{aligned}
$$

for $(m, n) \in I_{M^{\times}} J$, where

$$
c(M, n)=W(a(M, n))+b(M, n) \sum_{i=1}^{k} \sum_{s=m_{0}}^{M-1} \sum_{t=n_{0}}^{n-1} g_{i}(s, t) .
$$

Now, define a function $\Gamma(m, n)$ by the right-hand side of $(2.11)$. Clearly, $\Gamma(m, n)$ is positive and nondecreasing in each variable, with $\Gamma\left(m_{0}, n\right)=c(M, n)>0$. Hence (2.11) is equivalent to

$$
\eta(m, n) \leq W^{-1}(\Gamma(m, n))
$$

for all $(m, n) \in I_{M} \times J_{N_{1}}$, where $N_{1}$ is defined in (2.5). By (H4) and the monotonicity of $\phi, \psi^{-1}, W^{-1}$ and $\Gamma$, we have, for all $(m, n) \in I_{M} \times J_{N_{1}}$,

$$
\begin{aligned}
\Delta_{1} \Gamma(m, n) & =b(M, n) \sum_{i=1}^{k} \sum_{t=n_{0}}^{n-1} f_{i}(m, t) \varphi\left(\psi^{-1}(\eta(m, t))\right) \\
& \leq b(M, n) \varphi\left(\psi^{-1}\left(W^{-1}(\Gamma(m, n))\right)\right) \sum_{i=1}^{k} \sum_{t=n_{0}}^{n-1} f_{i}(m, t) .
\end{aligned}
$$

On the other hand, by the monotonicity of $\phi, \psi^{-1}$, and $W^{-1}$, we have

$$
\begin{aligned}
\Phi(\Gamma(m+1, n))-\Phi(\Gamma(m, n)) & =\int_{\Gamma(m, n)}^{\Gamma(m+1, n)} \frac{\mathrm{d} s}{\varphi\left(\psi^{-1}\left(W^{-1}(s)\right)\right)} \\
& \leq \frac{\Delta_{1} \Gamma(m, n)}{\varphi\left(\psi^{-1}\left(W^{-1}(\Gamma(m, n))\right)\right)} .
\end{aligned}
$$

From (2.14) and (2.15), we obtain

$$
\Phi(\Gamma(m+1, n))-\Phi(\Gamma(m, n)) \leq b(M, n) \sum_{i=1}^{k} \sum_{t=n_{0}}^{n-1} f_{i}(m, t)
$$

for $(m, n),(m+1, n) \in I_{M} \times J_{N_{1}}$. Keeping $n$ fixed and substituting $m$ with $s$ in (2.16), and then summing up both sides over $s$ from $m_{0}$ to $m-1$, we get

$$
\begin{aligned}
\Phi(\Gamma(m, n)) & \leq \Phi\left(\Gamma\left(m_{0}, n\right)\right)+b(M, n) \sum_{i=1}^{k} \sum_{s=m_{0}}^{m-1} \sum_{t=n_{0}}^{n-1} f_{i}(s, t) \\
& =\Phi(c(M, n))+b(M, n) \sum_{i=1}^{k} \sum_{s=m_{0}}^{m-1} \sum_{t=n_{0}}^{n-1} f_{i}(s, t)
\end{aligned}
$$


for $(m, n) \in I_{M} \times J_{N_{1}}$. From (2.12) and (2.17), we have

$$
\begin{aligned}
\Gamma(m, n) \leq & \Phi^{-1}\left(\Phi(c(M, n))+b(M, n) \sum_{i=1}^{k} \sum_{s=m_{0}}^{m-1} \sum_{t=n_{0}}^{n-1} f_{i}(s, t)\right) \\
= & \Phi^{-1}\left[\Phi\left(W(a(M, n))+b(M, n) \sum_{i=1}^{k} \sum_{s=m_{0}}^{M-1} \sum_{t=n_{0}}^{n-1} g_{i}(s, t)\right)\right. \\
& \left.+b(M, n) \sum_{i=1}^{k} \sum_{s=m_{0}}^{m-1} \sum_{t=n_{0}}^{n-1} f_{i}(s, t)\right] .
\end{aligned}
$$

From (2.7), (2.13), and (2.18), we get

$$
\begin{aligned}
& u(m, n) \leq \psi^{-1}(\eta(m, n)) \leq \psi^{-1}\left(W^{-1}(\Gamma(m, n))\right) \\
& \leq \psi^{-1}\left\{W ^ { - 1 } \left[\Phi^{-1}(\Phi(W(a(M, n))\right.\right. \\
& \left.+b(M, n) \sum_{i=1}^{k} \sum_{s=m_{0}}^{M-1} \sum_{t=n_{0}}^{n-1} g_{i}(s, t)\right) \\
& \left.\left.\left.+b(M, n) \sum_{i=1}^{k} \sum_{s=m_{0}}^{m-1} \sum_{t=n_{0}}^{n-1} f_{i}(s, t)\right)\right]\right\}
\end{aligned}
$$

for $(m, n) \in I_{M} \times J_{N_{1}}$. Let $m=M$, from (2.20), we observe that

$$
\begin{aligned}
u(M, n) \leq & \psi^{-1}\left\{W ^ { - 1 } \left[\Phi ^ { - 1 } \left(\Phi\left(W(a(M, n))+b(M, n) \sum_{i=1}^{k} \sum_{s=m_{0}}^{M-1} \sum_{t=n_{0}}^{n-1} g_{i}(s, t)\right)\right.\right.\right. \\
& \left.\left.\left.+b(M, n) \sum_{i=1}^{k} \sum_{s=m_{0}}^{M-1} \sum_{t=n_{0}}^{n-1} f_{i}(s, t)\right)\right]\right\}
\end{aligned}
$$

for all $(M, n) \in I_{M_{1}} \times J_{N_{1}}$, where $M_{1}$ is defined by (2.5). Since $M \in I_{M_{1}}$ is arbitrary, from (2.21), we get the required estimate

$$
\begin{aligned}
u(m, n) \leq & \psi^{-1}\left\{W ^ { - 1 } \left[\Phi ^ { - 1 } \left(\Phi\left(W(a(m, n))+b(m, n) \sum_{i=1}^{k} \sum_{s=m_{0}}^{m-1} \sum_{t=n_{0}}^{n-1} g_{i}(s, t)\right)\right.\right.\right. \\
& \left.\left.\left.+b(m, n) \sum_{i=1}^{k} \sum_{s=m_{0}}^{m-1} \sum_{t=n_{0}}^{n-1} f_{i}(s, t)\right)\right]\right\}
\end{aligned}
$$

for all $(m, n) \in I_{M_{1}} \times J_{N_{1}}$. Theorem 1 is proved.

Theorem 2. Suppose $\left(H_{1}-H_{5}\right)$ hold and $u(m, n)$ is a nonnegative function on $I \times J$ satisfying (1.2). Then

(i) if $\phi_{1}(u) \geq \phi_{2}(\log u)$, we have

$$
u(m, n) \leq \psi^{-1}\left[W^{-1}\left(\Phi_{1}^{-1}\left(D_{1}(m, n)\right)\right)\right]
$$

for all $(m, n) \in I_{M_{1}} \times J_{N_{2}}$,

(ii) if $\phi_{1}(u) \leq \phi_{2}(\log u)$, we have

$$
u(m, n) \leq \psi^{-1}\left[W^{-1}\left(\Phi_{2}^{-1}\left(D_{2}(m, n)\right)\right)\right]
$$


for all $(m, n) \in I_{M_{3}} \times J_{N_{3}}$ where

$$
\begin{aligned}
D_{j}(m, n): & =\Phi_{j}(W(a(m, n)))+b(m, n) \sum_{i=1}^{k} \sum_{s=m_{0}}^{m-1} \sum_{t=n_{0}}^{n-1}\left[f_{i}(s, t)+g_{i}(s, t)\right] ; \\
\Phi_{j}(r): & =\int_{1}^{r} \frac{\mathrm{d} s}{\varphi_{j}\left(\psi^{-1}\left(W^{-1}(s)\right)\right)} \text { for } r>0 ; \quad \Phi_{j}(0):=\lim _{r \rightarrow 0^{+}} \Phi_{j}(r) ;
\end{aligned}
$$

$j=1,2 ;\left(M_{2}, N_{2}\right)$ is arbitrarily given on the boundary of the planar region

$$
\mathcal{R}_{1}:=\left\{(m, n) \in I \times J: D_{1}(m, n) \in \operatorname{Dom}\left(\Phi_{1}^{-1}\right), \Phi_{1}^{-1}\left(D_{1}(m, n)\right) \in \operatorname{Dom}\left(W^{-1}\right)\right\} 2
$$

and $\left(M_{3}, N_{3}\right)$ is arbitrarily given on the boundary of the planar region

$$
\mathcal{R}_{2}:=\left\{(m, n) \in I \times J: D_{2}(m, n) \in \operatorname{Dom}\left(\Phi_{2}^{-1}\right), \Phi_{2}^{-1}\left(D_{2}(m, n)\right) \in \operatorname{Dom}\left(W^{-1}\right)\right\} 2
$$

Proof. (i) When $\phi_{1}(u) \geq \phi_{2}(\log u)$, from inequality (1.2), we have

$$
\begin{aligned}
\psi(u(m, n)) \leq & a(M, n)+b(M, n) \sum_{i=1}^{k} \sum_{s=m_{0}}^{m-1} \sum_{t=n_{0}}^{n-1} w\left(u\left(\alpha_{i}(s), \beta_{i}(t)\right)\right) \\
& \cdot\left[f_{i}(s, t) \varphi_{1}\left(u\left(\alpha_{i}(s), \beta_{i}(t)\right)\right)+g_{i}(s, t) \varphi_{2}\left(\log \left(u\left(\alpha_{i}(s), \beta_{i}(t)\right)\right)\right)\right]
\end{aligned}
$$

for all $(m, n) \in I_{M} \times J$, where $m_{0} \leq M \leq M_{2}$ is chosen arbitrarily. Let $\Xi(m, n)$ denote the right-hand side of (2.27), which is a positive and nondecreasing function in each variable with $\Xi\left(m_{0}, n\right)=a(M, n)$. Hence (2.27) is equivalent to

$$
u(m, n) \leq \psi^{-1}(\Xi(m, n)) .
$$

By $(H 4)$ and the monotonicity of $w, \psi^{-1}$, and $\Xi$, we have, for all $(m, n) \in I_{M} \times J$,

$$
\begin{aligned}
\Delta_{1} \Xi(m, n)= & b(M, n) \sum_{i=1}^{k} \sum_{t=n_{0}}^{n-1} w\left(u\left(\alpha_{i}(m), \beta_{i}(t)\right)\right) \\
& \cdot\left[f_{i}(m, t) \varphi_{1}\left(u\left(\alpha_{i}(m), \beta_{i}(t)\right)\right)+g_{i}(m, t) \varphi_{2}\left(\log \left(u\left(\alpha_{i}(m), \beta_{i}(t)\right)\right)\right)\right] \\
\leq & b(M, n) w\left(\psi^{-1}(\Xi(m, n))\right) \\
& \cdot \sum_{i=1}^{k} \sum_{t=n_{0}}^{n-1}\left[f_{i}(m, t) \varphi_{1}\left(\psi^{-1}(\Xi(m, t))\right)+g_{i}(m, t) \varphi_{2}\left(\log \left(\psi^{-1}(\Xi(m, t))\right)\right)\right]
\end{aligned}
$$

for all $(m, n) \in I_{M} \times J$. Similar to the process from (2.9) to (2.11), we obtain

$$
\begin{aligned}
W(\Xi(m, n)) \leq & W\left(\Xi\left(m_{0}, n\right)\right)+b(M, n) \sum_{i=1}^{k} \sum_{s=m_{0}}^{m-1} \sum_{t=n_{0}}^{n-1}\left[f_{i}(s, t) \varphi_{1}\left(\psi^{-1}(\Xi(s, t))\right)\right. \\
& \left.+g_{i}(s, t) \varphi_{2}\left(\log \left(\psi^{-1}(\Xi(s, t))\right)\right)\right] \\
= & W(a(M, n))+b(M, n) \sum_{i=1}^{k} \sum_{s=m_{0}}^{m-1} \sum_{t=n_{0}}^{n-1}\left[f_{i}(s, t) \varphi_{1}\left(\psi^{-1}(\Xi(s, t))\right)\right. \\
& \left.+g_{i}(s, t) \varphi_{2}\left(\log \left(\psi^{-1}(\Xi(s, t))\right)\right)\right] \\
\leq & W(a(M, n)) \\
& +b(M, n) \sum_{i=1}^{k} \sum_{s=m_{0}}^{m-1} \sum_{t=n_{0}}^{n-1}\left[f_{i}(s, t)+g_{i}(s, t)\right] \varphi_{1}\left(\psi^{-1}(\Xi(s, t))\right)
\end{aligned}
$$


for all $(m, n) \in I_{M} \times J$. Now, define a function $\Theta(m, n)$ by the right-hand side of (2.30). Clearly, $\Theta(m, n)$ is positive and nondecreasing in each variable, with $\Theta\left(m_{0}, n\right)=$ $W(a(M, n))>0$. Thus, $(2.30)$ is equivalent to

$$
\Xi(m, n) \leq W^{-1}(\Theta(m, n)) \quad \forall(m, n) \in I_{M} \times J_{N_{2}},
$$

where $N_{2}$ is defined by (2.25). Similar to the process from (2.14) to (2.18), we obtain

$$
\begin{aligned}
\Theta(m, n) & \leq \Phi_{1}^{-1}\left(\Phi_{1}\left(\Theta\left(m_{0}, n\right)\right)+b(M, n) \sum_{i=1}^{k} \sum_{s=m_{0}}^{m-1} \sum_{t=n_{0}}^{n-1}\left[f_{i}(s, t)+g_{i}(s, t)\right]\right) \\
& =\Phi_{1}^{-1}\left(\Phi_{1}(W(a(M, n)))+b(M, n) \sum_{i=1}^{k} \sum_{s=m_{0}}^{m-1} \sum_{t=n_{0}}^{n-1}\left[f_{i}(s, t)+g_{i}(s, t)\right]\right)^{(2.32}
\end{aligned}
$$

for all $(m, n) \in I_{M} \times J_{N_{2}}$. From (2.28), (2.31), and (2.32), we conclude that

$$
\begin{aligned}
u(m, n) & \leq \psi^{-1}(\Xi(m, n)) \leq \psi^{-1}\left(W^{-1}(\Theta(m, n))\right) \\
& \leq \psi^{-1}\left[W^{-1}\left(\Phi_{1}^{-1}\left(\Phi_{1}(W(a(M, n)))+b(M, n) \sum_{i=1}^{k} \sum_{s=m_{0}}^{m-1} \sum_{t=n_{0}}^{n-1}\left[f_{i}(s, t)+g_{i}(s, t)\right]\right)\right)\right]
\end{aligned}
$$

for all $(m, n) \in I_{M} \times J_{N_{2}}$. Let $m=M$, from (2.33), we get

$$
u(M, n) \leq \psi^{-1}\left[W^{-1}\left(\Phi_{1}^{-1}\left(\Phi_{1}\left(W(a(M, n))+b(M, n) \sum_{i=1}^{k} \sum_{s=m_{0}}^{M-1} \sum_{t=n_{0}}^{n-1}\left[f_{i}(s, t)+g_{i}(s, t)\right]\right)\right)\right] .\right.
$$

Since $M \in I_{M_{2}}$ is arbitrary, from inequality (2.34), we obtain the required inequality in (2.22).

(ii) When $\phi_{1}(u) \leq \phi_{2}(\log u)$, similar to the process from (2.27) to (2.30), from inequality (1.2), we have

$$
W(\Xi(m, n)) \leq W(a(M, n))+b(M, n) \sum_{i=1}^{k} \sum_{s=m_{0}}^{m-1} \sum_{t=n_{0}}^{n-1}\left[f_{i}(s, t)+g_{i}(s, t)\right] \varphi_{2}\left(\psi^{-1}(\Xi(s, t))\right)
$$

for all $(m, n) \in I_{M} \times J, M \in I_{M_{3}}$, where $M_{3}$ is defined in (2.26). Similar to the process from (2.30) to (2.34), we obtain

$$
u(M, n) \leq \psi^{-1}\left[W^{-1}\left(\Phi_{2}^{-1}\left(\Phi_{2}\left(W(a(M, n))+b(M, n) \sum_{i=1}^{k} \sum_{s=m_{0}}^{M-1} \sum_{t=n_{0}}^{n-1}\left[f_{i}(s, t)+g_{i}(s, t)\right]\right)\right)\right] .\right.
$$

Since $M \in I_{M_{3}}$ is arbitrary, from inequality (2.36), we obtain the required inequality in (2.23).

Theorem 3. Suppose $\left(H_{1}-H_{5}\right)$ hold and that $L, M \in C\left(\mathbb{R}_{+}^{3}, \mathbb{R}_{+}\right)$satisfy

$$
0 \leq L(s, t, u)-L(s, t, v) \leq M(s, t, v)(u-v)
$$

for $s, t, u, v \in \mathbb{R}_{+}$with $u>v \geq 0$. If $u(m, n)$ is a nonnegative function on $I \times J$ satisfying (1.3) then we have

$$
u(m, n) \leq \psi^{-1}\left[W^{-1}\left(\Phi_{3}^{-1}(E(m, n))\right)\right]
$$

for all $(m, n) \in I_{M_{4}} \times J_{N_{\psi}}$ where $W$ is defined by (2.2),

$$
\Phi_{3}(r):=\int_{1}^{r} \frac{\mathrm{d} s}{\psi^{-1}\left(W^{-1}(s)\right)} \text { for } r>0 ; \quad \Phi_{3}(0):=\lim _{r \rightarrow 0^{+}} \Phi_{3}(r),
$$




$$
\begin{aligned}
& E(m, n):=\Phi_{3}(F(m, n))+b(m, n) \sum_{i=1}^{k} \sum_{s=m_{0}}^{m-1} \sum_{t=n_{0}}^{n-1}\left[f_{i}(s, t) M(s, t, 0)+g_{i}(s, t)\right], \\
& F(m, n):=W(a(m, n))+b(m, n) \sum_{i=1}^{k} \sum_{s=m_{0}}^{m-1} \sum_{t=n_{0}}^{n-1} f_{i}(s, t) L(s, t, 0),
\end{aligned}
$$

and $\left(M_{4}, N_{4}\right) \in I \times J$ is arbitrarily given on the boundary of the planar region

$$
\mathcal{R}:=\left\{(m, n) \in I \times J: E(m, n) \in \operatorname{Dom}\left(\Phi_{3}^{-1}\right), \Phi_{3}^{-1}(E(m, n)) \in \operatorname{Dom}\left(W^{-1}\right)\right\} .
$$

Proof. From inequality (1.3), we have

$$
\begin{aligned}
\psi(u(m, n)) \leq & a(M, n)+b(M, n) \sum_{i=1}^{k} \sum_{s=m_{0}}^{m-1} \sum_{t=n_{0}}^{n-1} w\left(u\left(\alpha_{i}(s), \beta_{i}(t)\right)\right)\left[f_{i}(s, t) L\left(s, t, u\left(\alpha_{i}(s), \beta_{i}(t)\right)\right)\right. \\
& \left.+g_{i}(s, t) u\left(\alpha_{i}(s), \beta_{i}(t)\right)\right]
\end{aligned}
$$

for all $(m, n) \in I_{M} \times J$, where $m_{0} \leq M \leq M_{4}$ is chosen arbitrarily. Let $P(m, n)$ denote the right-hand side of (2.41), which is a positive and nondecreasing function in each variable, with $P\left(m_{0}, n\right)=a(M, n)$. Similar to the process in the proof of Theorem 2 from (2.27) to (2.30), we obtain

$$
\begin{aligned}
W(P(m, n)) \leq & W(a(M, n))+b(M, n) \sum_{i=1}^{k} \sum_{s=m_{0}}^{m-1} \sum_{t=n_{0}}^{n-1}\left[f_{i}(s, t) L\left(s, t, \psi^{-1}(P(s, t))\right)\right. \\
& \left.+g_{i}(s, t) \psi^{-1}(P(s, t))\right]
\end{aligned}
$$

for all $(m, n) \in I_{M} \times J$. From inequality (2.37) and (2.42), we get

$$
\begin{aligned}
W(P(m, n)) \leq & W(a(M, n))+b(M, n) \sum_{i=1}^{k} \sum_{s=m_{0}}^{m-1} \sum_{t=n_{0}}^{n-1} f_{i}(s, t) L(s, t, 0) \\
& +b(M, n) \sum_{i=1}^{k} \sum_{s=m_{0}}^{m-1} \sum_{t=n_{0}}^{n-1}\left[f_{i}(s, t) M(s, t, 0)+g_{i}(s, t)\right] \psi^{-1}(P(s, t))
\end{aligned}
$$

for all $(m, n) \in I_{M} \times J$. Similar to the process in the proof of Theorem 2 from (2.30) to $(2.34)$, we obtain

$$
\begin{aligned}
u(m, n) \leq & \psi^{-1}\left[W ^ { - 1 } \left(\Phi _ { 3 } ^ { - 1 } \left(\Phi_{3}\left(W(a(M, n))+b(M, n) \sum_{i=1}^{k} \sum_{s=m_{0}}^{m-1} \sum_{t=n_{0}}^{n-1} f_{i}(s, t) L(s, t, 0)\right)\right.\right.\right. \\
& \left.\left.\left.+b(m, n) \sum_{i=1}^{k} \sum_{s=m_{0}}^{m-1} \sum_{t=n_{0}}^{n-1}\left[f_{i}(s, t) M(s, t, 0)+g_{i}(s, t)\right]\right)\right)\right] .
\end{aligned}
$$

Since $M \in I_{M_{4}}$ is arbitrary, where $M_{4}$ is defined in (2.40), from inequality (2.43), we obtain the required inequality in (2.38).

\section{Applications to BVP}

In this section, we use our result to study certain properties of the solutions of the following boundary value problem (BVP):

$$
\left\{\begin{array}{l}
\Delta_{2}\left(\Delta_{1}(\psi(z(m, n)))\right)=F\left(m, n, z\left(\alpha_{1}(m), \beta_{1}(n)\right), z\left(\alpha_{2}(m), \beta_{2}(n)\right), \ldots, z\left(\alpha_{k}(m), \beta_{k}(n)\right)\right), \\
z\left(m, n_{0}\right)=a_{1}(m), z\left(m_{0}, n\right)=a_{2}(n) z\left(m, n_{0}\right)=a_{1}\left(m_{0}\right)=a_{2}\left(n_{0}\right)=0
\end{array}\right.
$$


for $m \in I, n \in J$, where $m_{0}, n_{0}, m_{1}, n_{1} \in \mathbb{R}_{+}$are constants, $I:=\left[m_{0}, m_{1}\right] \cap \mathbb{N}_{+}, J:=$ $\left[n_{0}, n_{1}\right] \cap \mathbb{N}_{+}, F: I \times J \times \mathbb{R}^{k} \rightarrow \mathbb{R}, \psi: \mathbb{R} \rightarrow \mathbb{R}$ is strictly increasing on $\mathbb{R}_{+}$with $\psi(0)=0$, $|\psi(r)|=\psi(|r|)$, and $\psi(t) \rightarrow \infty$ as $t \rightarrow \infty$; functions $\alpha_{i}: I \rightarrow I$ and $\beta_{i}: J \rightarrow J$ are nondecreasing such that $\alpha_{i}(m) \leq m$ and $\beta_{i}(n) \leq n, i=1,2, \ldots, k ;\left|a_{1}\right|: I \rightarrow \mathbb{R}_{+},\left|a_{2}\right|: J \rightarrow \mathbb{R}$ + are both nondecreasing.

We give an upper bound estimate for solutions of BVP (3.1).

Corollary 1. Consider BVP (3.1) and suppose that F satisfies

$$
\left|F\left(m, n, u_{1}, u_{2}, \ldots, u_{k}\right)\right| \leq \sum_{i=1}^{k} w\left(\left|u_{i}\right|\right)\left[f_{i}(m, n) \varphi\left(\left|u_{i}\right|\right)+g_{i}(m, n)\right], \quad(m, n) \in I \times J,
$$

where $f_{i}, g_{i}: I \times J \rightarrow \mathbb{R}_{+}$and $w, \phi \in C^{0}\left(\mathbb{R}_{+}, \mathbb{R}_{+}\right)$are nondecreasing with $w(u)>0, \phi(u)$ $>0$ for $u>0$. Then, all solutions $z(m, n)$ of $B V P$ (3.1) satisfy

$$
|z(m, n)| \leq \psi^{-1}\left(W^{-1}\left(\Phi^{-1}(A(m, n))\right)\right),
$$

for all $(m, n) \in I_{M_{1}} \times J_{N_{p}}$ where

$$
A(m, n):=\Phi\left(W\left(\psi\left(\left|a_{1}(m)\right|\right)+\psi\left(\left|a_{2}(n)\right|\right)\right)+\sum_{i=1}^{k} \sum_{s=m_{0}}^{m-1} \sum_{t=n_{0}}^{n-1} g_{i}(s, t)\right)+\sum_{i=1}^{k} \sum_{s=m_{0}}^{m-1} \sum_{t=n_{0}}^{n-1} f_{i}(s, t)
$$

for all $(m, n) \in I_{M_{1}} \times J_{N_{p}}$ with $W, W^{-1}, \Phi, \Phi^{-1}$ and $M_{1}, N_{1}$ as given in Theorem 1 .

Proof. BVP (3.1) is equivalent to

$$
\begin{aligned}
\psi(z(m, n))= & \psi\left(a_{1}(m)\right)+\psi\left(a_{2}(n)\right) \\
& +\sum_{s=m_{0}}^{m-1} \sum_{t=n_{0}}^{n-1} F\left(s, t, z\left(\alpha_{1}(s), \beta_{1}(t)\right), z\left(\alpha_{2}(s), \beta_{2}(t)\right), \ldots, z\left(\alpha_{k}(s), \beta_{k}(t)\right)\right)
\end{aligned}
$$

By (3.2) and (3.5), we get

$$
\begin{aligned}
& \psi(|z(m, n)|) \\
& \leq \psi\left(\left|a_{1}(m)\right|\right)+\psi\left(\left|a_{2}(n)\right|\right) \\
& \quad+\sum_{s=m_{0}}^{m-1} \sum_{t=n_{0}}^{n-1}\left|F\left(s, t, z\left(\alpha_{1}(s), \beta_{1}(t)\right), z\left(\alpha_{2}(s), \beta_{2}(t)\right), \ldots, z\left(\alpha_{k}(s), \beta_{k}(t)\right)\right)\right| \\
& \leq \psi\left(\left|a_{1}(m)\right|\right)+\psi\left(\left|a_{2}(n)\right|\right) \\
&+\sum_{s=m_{0}}^{m-1} \sum_{t=n_{0}}^{n-1} \sum_{i=1}^{k} w\left(\left|z\left(\alpha_{i}(s), \beta_{i}(t)\right)\right|\right)\left[f_{i}(s, t) \varphi\left(\left|z\left(\alpha_{i}(s), \beta_{i}(t)\right)\right|\right)+g_{i}(s, t)\right] .
\end{aligned}
$$

Clearly, inequality (3.6) is in the form of (1.1). Thus the estimate (3.3) of the solution $z(m, n)$ follows immediately from Theorem 1 .

\section{Acknowledgements}

The authors are very grateful to the editor and the referees for their helpful comments and valuable suggestions. This research was supported by National Natural Science Foundation of China(Project No. 11161018), Guangxi Natural Science Foundation(Project No. 0991265), and the Research Grants Council of the Hong Kong SAR, Project No. HKU7016/07P

\section{Author details}

${ }^{1}$ Department of Mathematics, Hechi University, Guangxi, Yizhou 546300, People's Republic of China ${ }^{2}$ School of Mathematics and Computing Science, Guilin University of Electronic Technology, Guilin 541004, People's Republic of China ${ }^{3}$ Department of Mathematics, The University of Hong Kong, Pokfulam Road, Hong Kong, People's Republic of China 
All the authors have contributed in all the paper part.

\section{Competing interests}

The authors declare that they have no competing interests.

\section{Received: 25 March 2011 Accepted: 10 October 2011 Published: 10 October 2011}

\section{References}

1. Bellman, R: The stability of solutions of linear differential equations. Duke Math J. 10, 643-647 (1943). doi:10.1215/S00127094-43-01059-2

2. Gronwall, TH: Note on the derivatives with respect to a parameter of the solutions of a system of differential equations. Ann Math. 20, 292-296 (1919). doi:10.2307/1967124

3. Agarwal, RP, Deng, S, Zhang, W: Generalization of a retarded Gronwall-like inequality and its applications. Appl Math Comput. 165, 599-612 (2005). doi:10.1016/j.amc.2004.04.067

4. Bainov, D, Simeonov, P: Integral Inequalities and Applications. Kluwer Academic, Dordrecht (1992)

5. Bihari, IA: A generalization of a lemma of Bellman and its application to uniqueness problem of differential equation. Acta Math Acad Sci Hung. 7, 81-94 (1956). doi:10.1007/BF02022967

6. Cheung, WS: Some discrete nonlinear inequalities and applications to boundary value problems for difference equations. J Differ Equ Appl. 10, 213-223 (2004). doi:10.1080/10236190310001604238

7. Dafermos, CM: The second law of thermodynamics and stability. Arch Rat Mech Anal. 70, 167-179 (1979)

8. Dannan, F: Integral inequalities of Gronwall-Bellman-Bihari type and asymptotic behavior of certain second order nonlinear differential equations. J Math Anal Appl. 108, 151-164 (1985). doi:10.1016/0022-247X(85)90014-9

9. Dragomir, SS, Kim, YH: Some integral inequalities for functions of two variables. Electr J Differ Equ. 2003(10):1-13 (2003)

10. Lipovan, O: A retarded Gronwall-like inequality and its applications. J Math Anal Appl. 252, 389-401 (2000). doi:10.1006/ jmaa.2000.7085

11. $\mathrm{Ma}, \mathrm{QH}$, Yang, EH: Some new Gronwall-Bellman-Bihari type integral inequalities with delay. Period Math Hung. 44, 225-238 (2002). doi:10.1023/A:1019600715281

12. Massalitina, EV: On the Perow integro-summable inequality for functions of two variables. Ukrainian Math J. 56, 1864-1872 (2004). doi:10.1007/s11253-005-0156-0

13. Medina, R, Pinto, M: On the asymptotic behavior of solutions of a class of second order nonlinear differential equations. J Math Anal Appl. 135, 399-405 (1988). doi:10.1016/0022-247X(88)90163-1

14. Mitrinović, DS, Pečarić, JE, Fink, AM: Inequalities Involving Functions and Their Integrals and Derivatives. Kluwer Academic, Dordrecht (1991)

15. Pachpatte, BG: Inequalities for Differential and Integral Equations. Academic Press, New York (1998)

16. Pinto, M: Integral inequalities of Bihari-type and applications. Funkcial Ekvac. 33, 387-430 (1990)

17. Cheung, WS: Some new nonlinear inequalities and applications to boundary value problems. Nonlinear Anal. 64 2112-2128 (2006). doi:10.1016/j.na.2005.08.009

18. Agarwal, RP, Kim, YH, Sen, SK: New retarded integral inequalities with applications. J Inequ Appl 2008, 15 (2008). Art. ID 908784

19. Chen, CJ, Cheung, WS, Zhao, D: Gronwall-Bellman-Type integral inequalities and applications to BVPs. J Inequ Appl 2009, 15 (2009). Art. ID 258569

20. Hull, TE, Luxemburg, WAJ: Numerical methods and existence theorem for ordinary differential equations. Numer Math. 2, 30-41 (1960). doi:10.1007/BF01386206

21. Pachpatte, BG, Deo, SG: Stability of discrete time systems with retarded argument. Utilitas Math. 4, 15-33 (1973)

22. Willett, D, Wong, JSW: On the discrete analogues of some generalizations of Gronwall's inequality. Monatsh Math. 69, 362-367 (1964)

23. Cheung, WS, Ren, J: Discrete non-linear inequalities and applications to boundary value problems. J Math Anal Appl. 319, 708-724 (2006). doi:10.1016/j.jmaa.2005.06.064

24. Wang, WS: A generalized sum-difference inequality and applications to partial Difference equations. Adv Differ Equ 2008, 12 (2008). Art. ID 695495

25. Wang, WS: Estimation on certain nonlinear discrete inequality and applications to boundary value problem. Adv Differ Equ 2009, 8 (2009). Art. ID 708587

26. Pachpatte, BG: On some fundamental integral inequalities and their discrete analogues. J Inequal Pure Appl Math. 2(2), Article 15 (2001)

doi:10.1186/1687-1847-2011-41

Cite this article as: Wang et al.: Some new nonlinear retarded sum-difference inequalities with applications. Advances in Difference Equations 2011 2011:41. 